\title{
HIGH-FIDELITY CFD SIMULATIONS OF MICROFLUIDIC DEVICES
}

\author{
M.M. Athavale, A.J. Przekwas \\ CFD Research Corporation, 3325 Triana Blvd., Huntsville, AL 35805
}

\begin{abstract}
Current state-of-the-art Computational Fluid Dynamics (CFD) techniques are applied to two microfluidic devices. Flow simulations in a bistable microfluidic amplifier and a micropump with dynamic valves were performed with CFD-ACE, an advanced CFD code. The bistable amplifier simulations showed good agreement with experiments in steady-state and switching modes and hysteresis behavior. Preliminary simulations of the flow in a micropump with dynamic valves are in progress and flow rectification is correctly predicted. An advanced, integrated, CAD environment for MEMS involving 3D geometry models/meshing, fluids/structures solver and visualization tools is proposed.
\end{abstract}

\section{INTRODUCTION}

Advances in the CFD techniques coupled with the speed and capabilities of the computer hardware has made it possible to use these techniques for complex flow problems with good confidence and fast tumaround times. The work reported here is a demonstration of use of CFD techniques to microfluidic devices. The eventual aim of the present effort is to develop a virtual computational environment (VCE) for multi-disciplinary analyses of microfluidic devices. It will contain virtual model "assembly tools", advanced analysis techniques, graphics driven menus for quick problem set-up and parallel processing capability to provide a powerful tool that can be used in the design and optimization process of microfluidic devices.

\section{CODE DESCRIPTION}

The simulations were performed using CFD-ACE, an advanced CFD code developed at CFDRC[1]. This code uses a finite-volume, pressure-based methodology for integration of the Navier-Stokes equations on body-fitted coordinate (BFC) grids. The code has an implicit, structured grid, multi-domain solution capability. The physical and numerical models include high order spatial and temporal discretization, selection of boundary types, turbulence models, and a moving grid algorithm. This code has been validated using a variety of benchmark, and experimental flow problems. Currently it is being applied to microfluidic device simulations.

\section{SIMULATION RESULTS}

\section{Bistable Wall Attachment Amplier}

This device was described by Vollmer et.al.[2], and can be used as a fluidic amplifier or as an actuator/switch. A description of the problem and grid used is shown in Figure 1. Working gas at supply pressure $\mathrm{P}_{s}$ is fed to the supply nozzle which forms a jet. Position of this jet is controlled by application of control pressure $\mathrm{P}_{c}$ at one of the control ports. The jet pressure at the output port is used

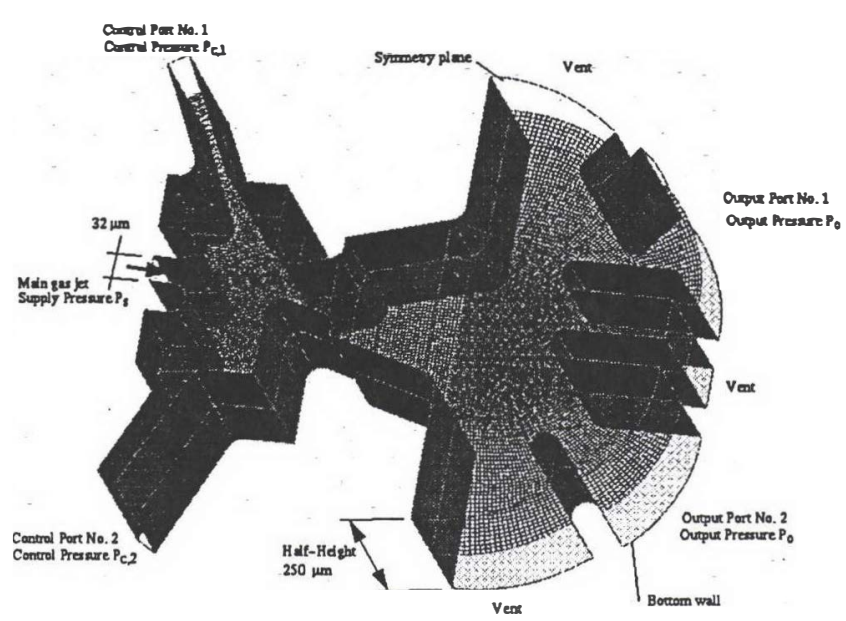

Figure 1. Flow domain, boundary conditions and computational grid for the amplifier.

to actuate or drive other micro-devices. The amplifier shows a hysteresis behavior during switching due to the Coanda effect at the walls as shown in Figure 2, where pressure $\mathrm{P}_{o}$ at the output port opposite to the control port is plotted as a function of $P_{c}$ in the increasing and decreasing mode.

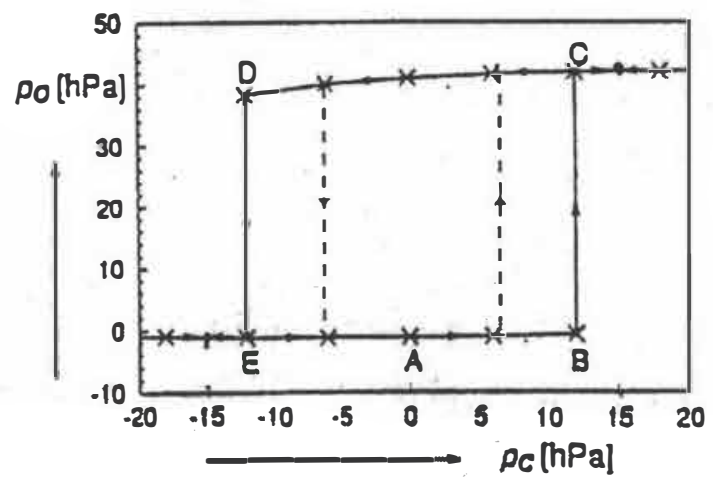

Figure 2. Swiching behavior of Bistable amplifier [2]. The dashed lines indicate the predicted switching behavior.

Simulation were performed to a) predict the steady-state jet at stable positions and calculate the output jet pressures, and b) vary $P_{c}$ in the hysteresis region to see if the observed hysteresis behavior is predicted. Both items are needed to give a successful demonstration result.

1. Problem Description: An 18-domain, 2-D, BFC structured grid, with $15 \mathrm{~K}$ cells was generated; the grid and boundary conditions are shown in Figure 1. The jet width was set at 32 microns. Nitrogen gas was the working fluid with density $=1.15 \mathrm{~kg} / \mathrm{m}^{3}$ and dynamic viscosity $=17.8 \mathrm{mPa}$-s. The supply pressure $\mathrm{P}_{s}$ was $33 \mathrm{hPa}$ (gage). The control pressure $P_{c}$ was applied at control port No. 2 (Figure 1), and all other ports were kept at $0 \mathrm{~Pa}$ (gage). The flow was assumed 
to be laminar and with constant fluxid properties.

1.2. Results of Simulations : Steady state jet results in terms of the horizontal velocities are shown in Figures $3 \mathrm{a}$ and $3 \mathrm{~b}$ where the $\mathrm{P}_{c}$ was set to $+/-15 \mathrm{hPa}$ to generate stable jets. The simulations used quiescent flow initial conditions. Comparison with Figure 2 shows that the jet positions are consistent with the experimental data. The maximum calculated jet pressure $\mathrm{P}_{\sigma}$ at the output port is $75 \mathrm{hPa}$, which compares with an experimental passage-average value of 40 $\mathrm{hPa}$. Two series of steady-state runs were performed next to simulate the switching behavior and hysteresis loop (Figure 2). These runs consisted of (a) series $\mathrm{A}$ where starting from the solution for $\mathrm{P}_{c}=15 \mathrm{hPa}$, the control pressure was decreased to $-12 \mathrm{hPa}$, and (b) series $\mathrm{B}$ where $\mathrm{P}_{c}=-15 \mathrm{hPa}$ at start and was increased to $+12 \mathrm{hPa}$. In both cases, the converged solution at the previous higher/lower $\mathrm{P}_{c}$ setting was used as the initial condition for the next run. Results of the series $A$ are shown in Figures $4 \mathrm{a}$ through $4 \mathrm{~d}$. The jet starts out exiting in output port No. 1 for $\mathrm{P}_{c}=15 \mathrm{hPa}$, and is seen to stay in that position till $\mathrm{P}_{c}$ is dropped to $-6 \mathrm{hPa}$; further lowering of $\mathrm{P}_{c}$ then moves the jet from the upper to lower output port. A symmetrical trend was seen in the series $B$ runs (results not shown) where $P_{c}$ was increased from $-15 \mathrm{hPa}$ to $12 \mathrm{hPa}$. The predicted switching pressures are between $+/-6 \mathrm{hPa}$ and $-1+12 \mathrm{hPa}$ which compare with $\mathrm{P}_{c}$ values of $+/-12 \mathrm{hPa}$ seen in Figure 2. Time-dependent simulations of the switching sequence also showed similar behavior.

\section{Micropump Simulations}

One class of micropumps use dynamic valves with diffuser shaped inlet/exit channels to provide a net fluid motion from inlet to exit. One such geometry was described by Gerlach and Wurmus [3] with high diffuser angles, and relies on flow separation in the diffusing direction to generate flow rectification.

2.1 Problem Description : A $7 \mathrm{~mm}$. square pump chamber with rectangular inlet and outlet sections with 70 degree diffuser section angles was used, with a deforming top wall. A 3-domain grid with 18000 cells was generated and a 'solids model' of the pump is shown

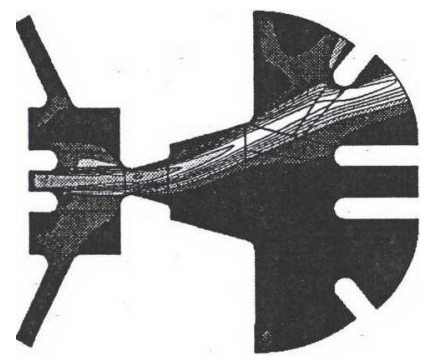

3a. $P_{c}=+15 \mathrm{hPa}$

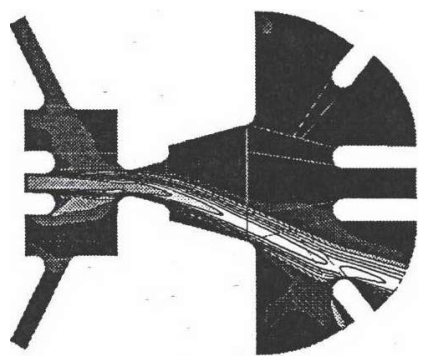

3b. $P_{c}=-15 \mathrm{hPa}$
Figure 3. Steady-State, Stable jet configurations. $P_{c}$ applied at the lower control port.

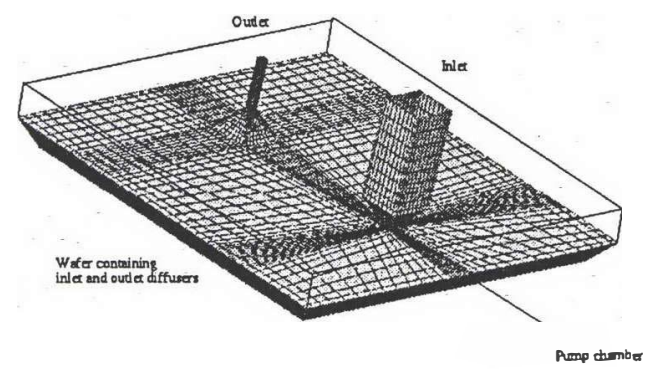

Figure 5. 'Solids model' of the micropump [3]. in Figure 5.

2.2 Simulation Results : Preliminary time-dependent simulations were carried out at a frequency of $3 \mathrm{KHz}$. The deformation amplitude is unknown, and needs to be matched with one experimental data point. After this anchoring, additional parametrics can be worked out. A typical velocity plot through the cross-section in the pump is shown in Figure 6, which shows the flow separation in the inlet diffuser during the pumping part of the cycle.

\section{CONCLUSIONS}

These simulations demonstrate the ability of CFD-ACE code to treat the complex geometries of the microfluidic devices considered here and provide reasonably accurate results of complex problem of the bistable amplifier. Additional validation problems and demonstration problems including simulations in microvalves are underway to establish the usefulness of the advanced CFD techniques in the analyses and designs of microfluidic devices.

This work was performed under ARPA/ETO SBIR Program and this support is greatly appreciated.

\section{REFERENCES} (1994).

1. CFD Research Corporation, "CFD-ACE User's Manual",

2. Vollmer, J., Hein, H., Menz, W., and Walter, F., "Bistable Fluidic Elements in LIGA Technique for Flow Control in Fluidic Microactuators", Sensors and Actuators A, (1994), Vol. 43, pp. 330334.

3. Gerlach, T., and Wurmus, H., "Working Principle and Performance of the Dynamic Micropump", Sensors and Actuators A, (1995), Vol.50, pp.135-140.

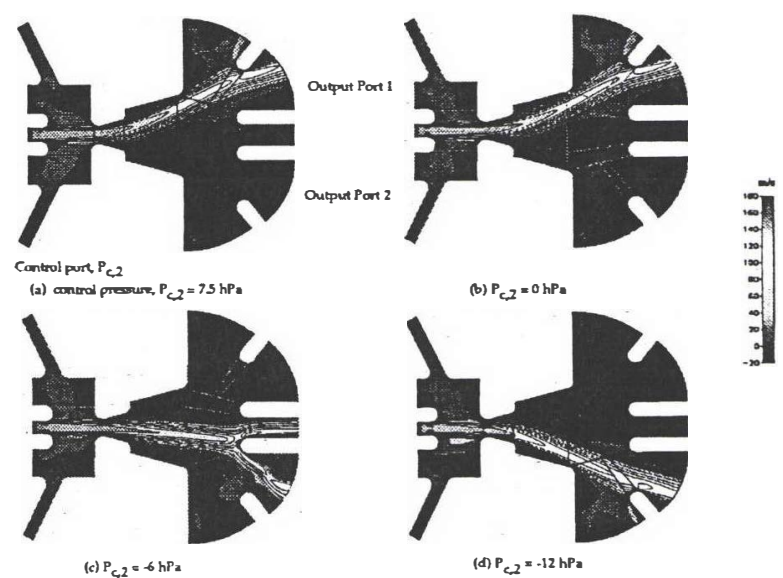

Figure 4. Dynamic Switching behavior of the amplifier, horizontal velocities.

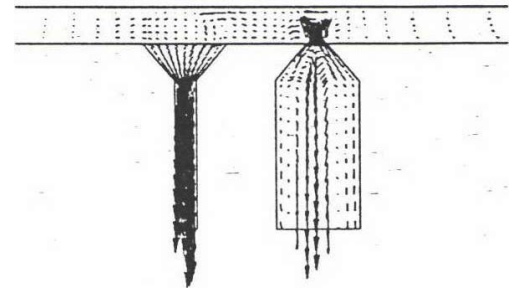

Figure 6. Velocity in pump cross-section during 'down-stroke'. 